OPEN ACCESS

Edited by:

Antonio Paoli,

University of Padua, Italy

Reviewed by:

Antonino Bianco,

University of Palermo, Italy

Patrik Drid,

University of Novi Sad, Serbia

${ }^{*}$ Correspondence:

Michal Wilk

m.wilk@awf.katowice.pl

Specialty section:

This article was submitted to

Exercise Physiology,

a section of the journal

Frontiers in Physiology

Received: 05 June 2020

Accepted: 20 October 2020

Published: 27 November 2020

Citation:

Wilk M, Gepfert M, Krzysztofik M, Stastny P, Zajac A and Bogdanis GC (2020) Acute Effects of Continuous

and Intermittent Blood Flow Restriction on Movement Velocity During Bench Press Exercise Against

Different Loads.

Front. Physiol. 11:569915.

doi: 10.3389/fphys.2020.569915

\section{Acute Effects of Continuous and Intermittent Blood Flow Restriction on Movement Velocity During Bench Press Exercise Against Different Loads}

\author{
Michal Wilk ${ }^{*}$, Mariola Gepfert ${ }^{1}$, Michal Krzysztofik ${ }^{1}$, Petr Stastny ${ }^{2}$, Adam Zajac ${ }^{1}$ and \\ Gregory C. Bogdanis ${ }^{3}$
}

${ }^{1}$ Institute of Sport Sciences, Jerzy Kukuczka Academy of Physical Education in Katowice, Katowice, Poland, ${ }^{2}$ Department of Sport Games, Faculty of Physical Education and Sport, Charles University, Prague, Czechia, ${ }^{3}$ School of Physical Education and Sport Science, National and Kapodistrian University of Athens, Athens, Greece

This study evaluated the effects of continuous and intermittent blood flow restriction (BFR) with $70 \%$ of full arterial occlusion pressure on bar velocity during the bench press exercise against a wide range of resistive loads. Eleven strength-trained males (age: $23.5 \pm 1.4$ years; resistance training experience: $2.8 \pm 0.8$ years, maximal bench press strength $-1 \mathrm{RM}=101.8 \pm 13.9 \mathrm{~kg}$; body mass $=79.8 \pm 10.4 \mathrm{~kg}$ ), performed three different testing protocols in random and counterbalanced order: without BFR (NOBFR); intermittent BFR (I-BFR) and continuous BFR (C-BFR). During each experimental session, subjects performed eight sets of two repetitions each, with increasing loads from 20 to 90\% 1RM (10\% steps), and 3 min rest between each set. In the C-BFR condition occlusion was kept throughout the trial, while in the I-BFR, occlusion was released during each 3 min rest interval. Peak bar velocity (PV) during the bench press exercise was higher by $12-17 \%$ in both I-BFR and C-BFR compared with NO-BFR only at the loads of $20,30,40$, and 50\% 1RM $(p<0.001)$, while performance at higher loads remained unchanged. Mean bar velocity (MV) was unaffected by occlusion $(p=0.342)$. These results indicate that BFR during bench press exercise increases PV and this may be used as an enhanced stimulus during explosive resistance training. At higher workloads, bench press performance was not negatively affected by BFR, indicating that the benefits of exercise under occlusion can be obtained while explosive performance is not impaired.

Keywords: occlusion, resistance exercise, cuff, peak velocity, performance

\section{INTRODUCTION}

Athletes as well as recreationally trained individuals are increasingly looking for innovative techniques and methods of resistance training to provide an additional stimulus to break through plateaus, prevent monotony and achieve various training goals (Krzysztofik et al., 2019). Partial or total occlusion of blood flow to the working muscles during resistance exercise, also known 
as blood flow restriction (BFR), has been used as a complementary training modality, aiming to further increase muscle mass and improve strength (Wilk et al., 2018).

Typically, BFR training involves the use of a tourniquet, an inflatable cuff, or elastic wraps that exert high pressure at the proximal part of the limb (arm or leg), in order to reduce arterial blood flow and to shut venous blood flow during exercise (Takano et al., 2005; Loenneke and Pujol, 2009; Scott et al., 2015). There are different modalities of BFR training, such as continuous BFR during a resistance training set or several sets, intermittent BFR used only during exercise, with release of occlusion during the rest interval, or even occlusion only before exercise, in order to induce ischemic preconditioning (Incognito et al., 2016; Marocolo et al., 2016, 2017, 2018, 2019; Wilk et al., 2020d,e,f). Resistance exercise under BFR affects physiological responses, increases mechanical tension, metabolic stress (Pearson and Hussain, 2015; Teixeira E. L. et al., 2018), cell swelling (Loenneke et al., 2012b), and enhances responses of the endocrine system (Takano et al., 2005; Shimizu et al., 2016). Individual limb characteristics, as well as the width, length, shape and material of the cuffs determine the level of applied pressure and as a consequence, modify post-exercise adaptive responses to BFR training (Loenneke et al., 2012b, 2015; Jessee et al., 2016; Rawska et al., 2019; Wilk et al., 2020e). However, not only physiological, but also mechanical factors related to BFR should be taken into consideration (Wilk et al., 2020d,f). Wilk et al. (2020f) speculated that mechanical work generated by the cuff, in addition to other factors, can potentially augment bar velocity and power output during resistance exercise under BFR. The BFR cuff is a passive element, but during exercise, the strain of the material and the deformation of the cuff may store and return elastic energy, which could affect power output and bar velocity compared to standard conditions (Wilk et al., 2020f).

Despite the fact much attention has been focused on BFR training, only few previous studies have compared the acute effects of BFR during resistance exercise (Luebbers et al., 2014; Teixeira E. L. et al., 2018; Rawska et al., 2019), and only one study has examined its effects on subsequent power output generation (Wilk et al., 2020f). Wilk et al. (2020f) showed that intermittent, high pressure BFR increases bar velocity and power output during the bench press (BP) exercise at $70 \%$ one-repetition maximum (1RM). However, only BFR with a wide cuff $(10 \mathrm{~cm})$ significantly influenced power output and bar velocity, while no changes were registered with a narrow cuff $(4 \mathrm{~cm})$ (Wilk et al., 2020f). As suggested by Wilk et al. (2020f) such potential benefits related to power output and bar velocity can be obtained only under high pressure BFR, but it is still unknown whether these acute gains are influenced by the mode of BFR (e.g., intermittent vs. continuous) or by the magnitude of external load used, relative to maximum strength. Thus, the impact of BFR on the velocity of movement during resistance exercise requires further research.

The BP is one of the most popular upper-body resistance exercises, with numerous variations (e.g., flat, incline, decline, wide-grip, and closed-grip) commonly used in practice (Dunnick et al., 2015; Wilk et al., 2019, 2020c). Given the widespread use of the BP as a basic exercise for developing upper body strength and power output (Stastny et al., 2017), it would be interesting to examine whether the use of continuous or intermittent BFR acutely affects bar velocity during the BP. Furthermore, it would be very useful from a practical viewpoint to determine whether the acute effects of BFR training are modified by the external load used. Therefore, the aim of the present study was to evaluate the effects of continuous and intermittent BFR on bar velocity during the BP exercise at progressive loads, from 20 to $90 \% 1 \mathrm{RM}$. It was hypothesized that both continuous and intermittent BFR would increase bar velocity during the BP at all used loads.

\section{MATERIALS AND METHODS}

The experiment was performed following a randomized crossover design, where each subject performed three different testing protocols in random and counterbalanced order, 1 week apart: without BFR (NO-BFR); intermittent BFR (I-BFR) and continuous BFR (C-BFR). Before the main tests, three familiarization session were performed. One week before the first main session, maximal BP strength (one repetition maximum1RM) was evaluated. During each experimental session, subjects performed eight sets of two repetitions each, with increasing loads from 20 to $90 \% 1 \mathrm{RM}$ (10\% steps), and $3 \mathrm{~min}$ rest between each set. Each repetition was performed with a $2 \mathrm{~s}$ duration of the eccentric phase of the movement and a maximal tempo in the concentric phase of the BP exercise. In the C-BFR condition occlusion was kept throughout the trial, while in the I-BFR, occlusion was released during each 3 min rest interval (Figure 1). The following variables were measured using a linear position transducer: PV and MV. All testing sessions were performed in the Strength and Power Laboratory at the Academy of Physical Education in Katowice, Poland.

\section{Subjects}

Power analysis indicated a minimum sample size of nine participants would be needed to detect an effect size (ES) of 0.44 . This value was obtained from a recent study examining the acute effects of BFR on movement velocity during the bench press (Wilk et al., 2020d). Power analysis was performed using the following parameters: type of analysis was set to repeated measures ANOVA, the required power was set to 0.80 , alpha was set to 0.05 , and the correlation between repeated measures was set to $r=0.5$ (G*Power software, v.3.1.9.2).

Eleven healthy men with experience in resistance training $(2.8 \pm 0.8$ years $)$ volunteered for the study after completing an informed consent form (age $=23.5 \pm 1.4$ years; body mass $=79.8 \pm 10.4 \mathrm{~kg} ; \mathrm{BP} 1 \mathrm{RM}=101.8 \pm 13.9 \mathrm{~kg})$. The main inclusion criteria were: a BP personal best of at least $120 \%$ body mass and that the subject was free from musculoskeletal injuries for at least 6 months before the study. The subjects were instructed to maintain their normal dietary habits over the course of the study for the duration of the experiment. The participants were instructed to maintain their usual hydration and dietary habits and not to use any supplements or stimulants during the study period and registering their calorie intake using "MyFitnessPal" software (Teixeira V. et al., 2018) every $24 \mathrm{~h}$ before the testing procedure. Compliance of these variables was verified at the start of each visit, before any data collection. They were informed about the benefits and potential risks of 


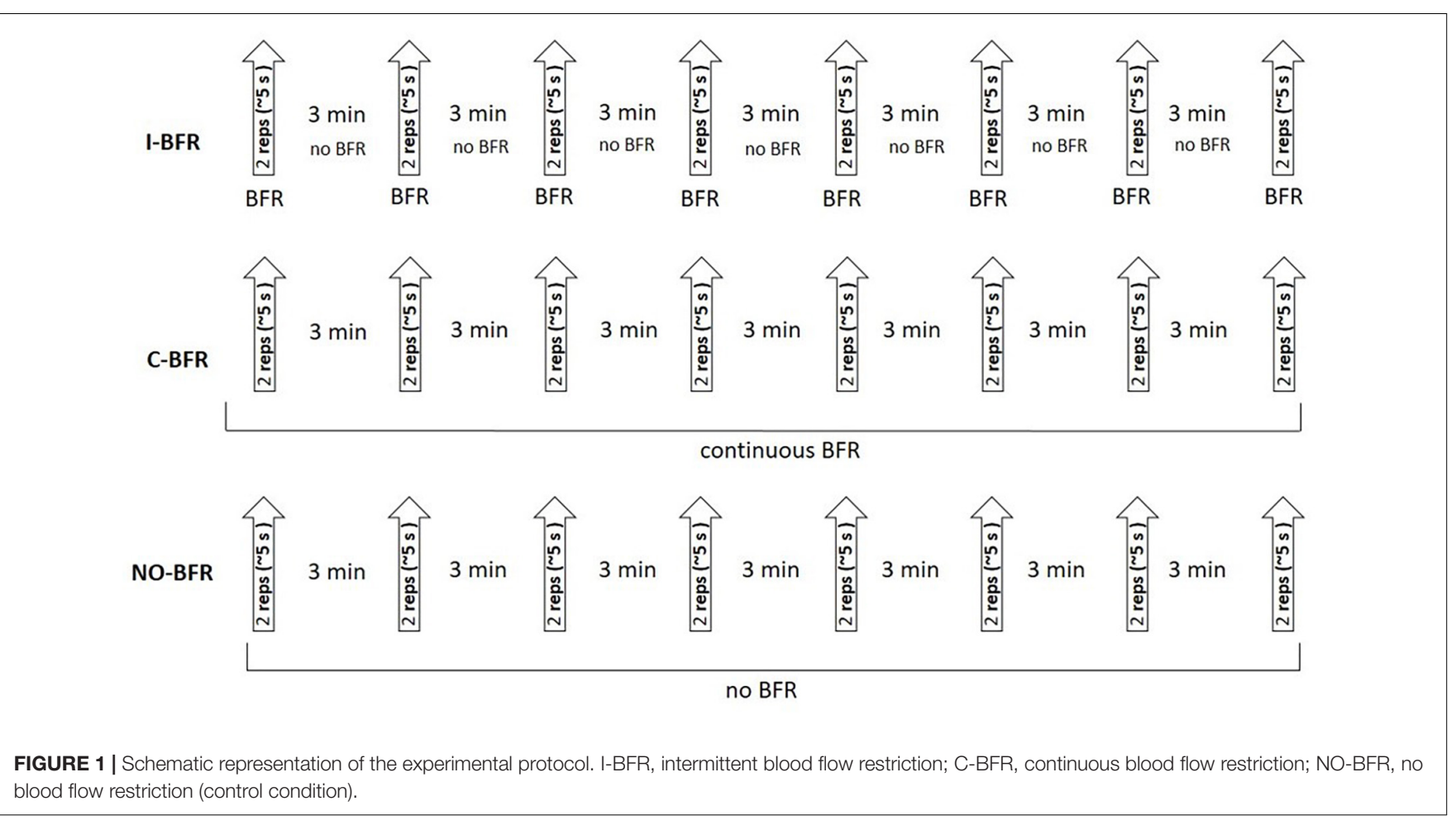

the study before providing their written informed consent for participation, and were allowed to withdraw from the study at any time. The study protocol was approved by the Bioethics Committee for Scientific Research, at the Academy of Physical Education in Katowice, Poland (02/2019), and all procedures were in accordance with the ethical standards of the Declaration of Helsinki, 1983.

\section{Procedures}

\section{Familiarization Session and the 1RM Strength Test}

Two weeks before the main experiment, the subjects performed at three familiarization sessions. During the familiarization sessions, each subject performed five sets of two repetitions of the BP under BFR against a load of 30,50 , and $70 \%$ of their estimated 1RM. The familiarization sessions were performed in order to minimize possible learning effects during the main tests. One week before the main experiment the 1RM BP test was performed. On arrival, body mass was measured and then the subjects cycled on a cycle-ergometer for $5 \mathrm{~min}$, followed by a general upper body warm-up as described elsewhere (Wilk et al., 2019). Then, the subjects performed 15, 10, and 5 BP repetitions using 20,40 , and $60 \%$ of their estimated $1 \mathrm{RM}$. The first testing load was set to an estimated $80 \% 1 \mathrm{RM}$, and was increased by $2.5-10 \mathrm{~kg}$ for each subsequent attempt. This process was repeated until failure. During the 1RM test, the subjects executed one repetition with $2 \mathrm{~s}$ of duration in the eccentric phase and maximal speed in the concentric phase of movement. According to guidelines by Wilk et al. (2020a,b) the 1RM test was performed with the same tempo of movement as all experimental trials. The rest interval between successful trials was $5 \mathrm{~min}$. Hand placement on the barbell was set at $150 \%$ of the individual bi-acromial distance, and this was used for all main trials.

\section{Experimental Sessions}

In a randomized and counterbalanced order, the subjects performed the BP exercise under three different testing conditions: without BFR (NO-BFR); intermittent BFR (I-BFR) and continuous BFR (C-BFR). During each testing protocol the subject performed the BP against an individualized load, starting from 20 to $90 \% 1 \mathrm{RM}$, which was increased progressively by $10 \%$ in each subsequent set (for a total of eight sets) with a $3 \mathrm{~min}$ rest interval between sets. During each set the subjects performed two repetitions, with a $2 \mathrm{~s}$ duration of the eccentric phase and maximal velocity in the concentric phase of movement. Every repetition was performed without bouncing the barbell off the chest, and without intentionally pausing at the transition between the eccentric and concentric phases. A linear position transducer system (Tendo Power Analyzer, Tendo Sport Machines, Trencin, Slovakia) was used for the evaluation of bar velocity (GarnachoCastaño et al., 2015). Measurements were made independently for each repetition and automatically converted into values of bar velocity. PV was obtained from the best repetition performed in a particular set. The MV was obtained as the mean of two repetitions performed in particular sets. All subjects completed the described testing protocol that was carefully replicated in subsequent experimental sessions.

\section{Blood Flow Restriction}

During the BFR sessions, subjects wore pressure cuffs at the most proximal region of both arms. For this experiment we used KAATSU cuffs (Master, Sato Sports Plaza, Tokyo, Japan), 
which are characterized as "narrow" $4-\mathrm{cm}$ cuffs (Wilk et al., 2020f). To determine the individual pressure value, after a $5 \mathrm{~min}$ rest interval, the value of full arterial occlusion pressure was determined. The measurement was conducted twice on each limb and the obtained differences were within $20 \mathrm{mmHg}$, with the average value used to set the cuff pressure for the exercise protocol (Wilk et al., 2020f). The cuff pressure for the BP exercise was set to $\sim 70 \%$ of full arterial occlusion pressure $(231 \pm 20 \mathrm{mmHg})$. The level of vascular restriction was monitored using a handheld Edan SD3 Doppler with an OLED screen and a $2 \mathrm{mHz}$ probe made by Edan Instruments (Shenzhen, China). For the I-BFR protocol, the occlusion was applied immediately before the start of the exercise and released upon completion of the second repetition. Thus, in the I-BFR condition, the occlusion was released during each $3 \mathrm{~min}$ rest interval. The occlusion for I-BFR lasted approximately $40 \mathrm{~s}$ (eight sets, $\sim 5 \mathrm{~s}$ of effort for each set). For the C-BFR condition the occlusion was applied $1 \mathrm{~min}$ before the start of the first set of the BP, and was maintained for all experimental sets, and also during the rest intervals. The occlusion for C-BFR lasted approximately $23 \mathrm{~min}$ (eight sets, $3 \mathrm{~min}$ rest intervals, $\sim 5 \mathrm{~s}$ of effort for each set).

\section{Statistical Analysis}

All statistical analysis were performed using Statistica 9.1. Results are presented as means with standard deviations. The ShapiroWilk, Levene, and Mauchly's tests were used in order to verify the normality, homogeneity and sphericity of the sample data variances, respectively. Differences between the NO-BFR, I-BFR and $\mathrm{C}-\mathrm{BFR}$ conditions were examined using repeated measures two-way ANOVA ( 3 conditions $\times 8$ loads). The statistical significance was set at $p<0.05$. ESs for main effects and interactions were determined by partial eta squared $\left(\eta^{2}\right)$. Partial eta squared values were classified as small (0.01-0.059), moderate (0.06-0.137) and large (>0.137). Post hoc comparisons using the Tukey's test were conducted to locate the differences between mean values when a main effect or an interaction was found. For pairwise comparisons, ESs were determined by Cohen's $d$ which was characterized as large $(d>0.8)$, moderate ( $d$ between 0.8 and 0.5 ), small ( $d$ between 0.49 and 0.20$)$ and trivial $(d<0.2)$ (Cohen, 1988). Percent changes with $90 \%$ confidence intervals (90CI) were also calculated. Statistical significance was set at $p<0.05$.

\section{RESULTS}

The two-way repeated measures ANOVA showed statistically significant interaction for PV (conditions $\times$ load; $p<0.0001$; $\eta^{2}=0.33$; Table 1). The post hoc analysis revealed that PV was higher by $12-17 \%$ in both I-BFR and C-BFR compared with NO-BFR at the loads of 20, 30, 40, and 50\% 1RM (Table 1 and Figure 2). For example, at the lowest load (20\% 1RM), PV in the NO-BFR condition was $2.01 \pm 0.49 \mathrm{~m} / \mathrm{s}$ (90CI; $1.77-2.25 \mathrm{~m} / \mathrm{s}$ ), and was increased to $2.34 \pm 0.34 \mathrm{~m} / \mathrm{s}$ (90CI; $2.17-2.51 \mathrm{~m} / \mathrm{s}$; $p<0.0001 ; d=0.82)$ in the I-BFR and to $2.33 \pm 0.33 \mathrm{~m} / \mathrm{s}(90 \mathrm{CI}$; $2.17-2.49 \mathrm{~m} / \mathrm{s} ; p<0.0001 ; d=0.80)$ in the C-BFR condition. At the load of $50 \% 1 \mathrm{RM}, \mathrm{PV}$ in the NO-BFR condition was $1.18 \pm 0.23 \mathrm{~m} / \mathrm{s}(90 \mathrm{CI} ; 1.07-1.29 \mathrm{~m} / \mathrm{s})$, and was increased to
$1.37 \pm 0.18 \mathrm{~m} / \mathrm{s}(90 \mathrm{CI} ; 1.28-1.46 \mathrm{~m} / \mathrm{s} ; p<0.0001 ; d=1.04)$ in the I-BFR and to $1.38 \pm 0.17 \mathrm{~m} / \mathrm{s}$ (90CI; $1.30-1.46 \mathrm{~m} / \mathrm{s} ; p<0.0001$; $d=0.80)$ in the C-BFR condition. No differences were found between the I-BFR and C-BFR conditions at any load.

There was also a main effect for condition for PV $(p=0.001$; $\left.\eta^{2}=0.50\right)$. The post hoc analysis showed that PV during both $\mathrm{C}-\mathrm{BFR}$ and I-BFR conditions was higher compared to NO-BFR ( $p=0.002$ and $p=0.004$, respectively).

There was no significant condition $\times$ load interaction $(p=0.342)$ or main effect $(p=0.871)$ for conditions for MV (Table 2).

\section{DISCUSSION}

The main finding of the study was that both intermittent and continuous BFR significantly increased PV during the BP exercise for lighter loads (20-50\% 1RM) but not for higher loads 60$90 \%$ 1RM, partially confirming our hypothesis. Furthermore, the results of the present study did not reveal any significant influence of BFR on MV at any load. Therefore, the results of this study indicate that BFR using a narrow cuff, commonly used for this type of training (KAATSU cuff) enhance peak velocity of the bar when the load is light, while they have no negative effect on PV or MV at any load, thus allowing the use of a wide range of resistances, without performance reduction in the first two repetitions.

Currently, only one previous study has analyzed the acute impact of BFR on bar velocity during the BP exercise, using a single load of 70\% $1 \mathrm{RM}$ and two types of cuffs: a wider $10 \mathrm{~cm}$ cuff and a narrow $4 \mathrm{~cm}$ cuff (Wilk et al., 2020f), identical to that used in the present study. The findings of study Wilk et al. (2020f) showed that bar velocity was increased but only when the wide cuff was used, while no changes in any BP performance were seen when the narrow cuff was used at 70\% 1RM. In that respect, the results of the present study, are consistent with the findings of that study regarding the load 70\% $1 \mathrm{RM}$ and the cuff width used. As previously suggested, the acute improvement of performance may be obtained only when wider BFR cuffs with high pressure are used during exercise (Wilk et al., 2020d), but it is unknown whether this effect is purely mechanical or if it also involves other physiological acute responses (Loenneke J. et al., 2012; Rossow et al., 2012). In the present study PV, but not MV, was enhanced in the lower resistive loads (up to 50\% 1RM), despite the fact that the cuff was narrow and cuff pressure was set to $70 \%$ AOP.

The lack of performance enhancement when resistance was $\geq 60 \% 1 \mathrm{RM}$, may be explained by the possibility of an equally compromised blood flow during exercise and rest interval in all conditions, irrespective of external pressure. When lifting loads at or above $60 \% 1 \mathrm{RM}$, intramuscular pressure is high due to muscle tension, and thus blood flow during exercise is occluded (Sjøgaard et al., 1988; Golas et al., 2015). Also, blood flow during recovery in the latter four sets in all trials, may be compromised due to muscle swelling, as a result of osmotic changes and fluid shifts into the muscle (Sjøgaard et al., 1988; Ploutz-Snyder et al., 1995; Bell et al., 2020). Thus, if blood flow during exercise and recovery is reduced due to muscle tension and muscle swelling, 
TABLE 1 | Peak bar velocity during the bench press exercise for eight different loads.

\begin{tabular}{|c|c|c|c|c|c|c|c|c|c|}
\hline & \multicolumn{8}{|c|}{ Load (\% 1RM) } & \multirow[b]{3}{*}{$p$ (condition $\times$ load) } \\
\hline & $20 \%$ 1RM & $30 \%$ 1RM & $40 \%$ 1RM & $50 \%$ 1RM & $60 \%$ 1RM & 70\% 1RM & $80 \%$ 1RM & $90 \% 1 R M$ & \\
\hline \multicolumn{9}{|c|}{ Peak bar velocity $(\mathrm{m} / \mathrm{s})$} & \\
\hline NO-BFR & $\begin{array}{c}2.01 \pm 0.49 \\
(1.77-2.25)\end{array}$ & $\begin{array}{l}1.77 \pm 0.44 \\
(1.55-1.99)\end{array}$ & $\begin{array}{l}1.41 \pm 0.27 \\
(1.28-1.54)\end{array}$ & $\begin{array}{c}1.18 \pm 0.23 \\
(1.07-1.29)\end{array}$ & $\begin{array}{l}0.97 \pm 0.17 \\
(0.89-1.05)\end{array}$ & $\begin{array}{c}0.86 \pm 0.20 \\
(0.76-0.96)\end{array}$ & $\begin{array}{c}0.61 \pm 0.13 \\
(0.55-0.67)\end{array}$ & $\begin{array}{c}0.39 \pm 0.15 \\
(0.32-0.46)\end{array}$ & 0.0001 \\
\hline I-BFR & $\begin{array}{c}2.34 \pm 0.34 \\
(2.11-2.57)\end{array}$ & $\begin{array}{l}1.99 \pm 0.20 \\
(1.86-2.13)\end{array}$ & $\begin{array}{l}1.61 \pm 0.21 \\
(1.47-1.75)\end{array}$ & $\begin{array}{l}1.37 \pm 0.18 \\
(1.25-1.49)\end{array}$ & $\begin{array}{l}1.02 \pm 0.23 \\
(0.86-1.17)\end{array}$ & $\begin{array}{c}0.84 \pm 0.16 \\
(0.73-0.94)\end{array}$ & $\begin{array}{c}0.67 \pm 0.14 \\
(0.57-0.76)\end{array}$ & $\begin{array}{c}0.46 \pm 0.14 \\
(0.37-0.56)\end{array}$ & \\
\hline C-BFR & $\begin{array}{c}2.33 \pm 0.33 \\
(2.17-2.49)\end{array}$ & $\begin{array}{l}1.98 \pm 0.19 \\
(1.89-2.07)\end{array}$ & $\begin{array}{l}1.60 \pm 0.20 \\
(1.50-1.70)\end{array}$ & $\begin{array}{l}1.38 \pm 0.17 \\
(1.30-1.46)\end{array}$ & $\begin{array}{l}1.00 \pm 0.21 \\
(0.90-1.10)\end{array}$ & $\begin{array}{c}0.83 \pm 0.15 \\
(0.76-0.90)\end{array}$ & $\begin{array}{c}0.66 \pm 0.14 \\
(0.59-0.73)\end{array}$ & $\begin{array}{c}0.44 \pm 0.14 \\
(0.37-0.51)\end{array}$ & \\
\hline
\end{tabular}

Results are mean $\pm S D$ (90\% confidence intervals). 1RM, 1 repetition maximum; NO-BFR, no blood flow restriction (control); I-BFR, intermittent blood flow restriction; C-BFR, continuous blood flow restriction.

TABLE 2 | Mean bar velocity during the bench press exercise for eight different loads.

\begin{tabular}{|c|c|c|c|c|c|c|c|c|c|}
\hline & \multicolumn{8}{|c|}{ Load (\% 1RM) } & \multirow[b]{3}{*}{$p$ (condition $\times$ load) } \\
\hline & $20 \%$ 1RM & $30 \%$ 1RM & $40 \%$ 1RM & $50 \%$ 1RM & $60 \%$ 1RM & $70 \%$ 1RM & $80 \%$ 1RM & $90 \% 1 \mathrm{RM}$ & \\
\hline & \multicolumn{8}{|c|}{ Mean bar velocity $(\mathrm{m} / \mathrm{s})$} & \\
\hline NO-BFR & $\begin{array}{l}1.41 \pm 0.24 \\
(1.29-1.53)\end{array}$ & $\begin{array}{l}1.22 \pm 0.14 \\
(1.15-1.29)\end{array}$ & $\begin{array}{l}1.05 \pm 0.12 \\
(0.99-1.11)\end{array}$ & $\begin{array}{c}0.90 \pm 0.08 \\
(0.86-0.94)\end{array}$ & $\begin{array}{l}0.70 \pm 0.11 \\
(0.65-0.75)\end{array}$ & $\begin{array}{c}0.55 \pm 0.10 \\
(0.50-0.60)\end{array}$ & $\begin{array}{c}0.39 \pm 0.08 \\
(0.35-0.43)\end{array}$ & $\begin{array}{c}0.24 \pm 0.04 \\
(0.22-0.26)\end{array}$ & 0.342 \\
\hline I-BFR & $\begin{array}{l}1.40 \pm 0.17 \\
(1.32-1.48)\end{array}$ & $\begin{array}{l}1.25 \pm 0.12 \\
(1.19-1.31)\end{array}$ & $\begin{array}{l}1.02 \pm 0.09 \\
(0.98-1.06)\end{array}$ & $\begin{array}{c}0.86 \pm 0.07 \\
(0.83-0.89)\end{array}$ & $\begin{array}{c}0.72 \pm 0.06 \\
(0.69-0.75)\end{array}$ & $\begin{array}{c}0.64 \pm 0.08 \\
(0.60-0.68)\end{array}$ & $\begin{array}{c}0.40 \pm 0.07 \\
(0.37-0.43)\end{array}$ & $\begin{array}{c}0.23 \pm 0.06 \\
(0.20-0.26)\end{array}$ & \\
\hline C-BFR & $\begin{array}{c}1.47 \pm 0.17 \\
(1.39-1.5)\end{array}$ & $\begin{array}{l}1.28 \pm 0.13 \\
(1.22-1.34)\end{array}$ & $\begin{array}{l}1.06 \pm 0.10 \\
(1.01-1.11)\end{array}$ & $\begin{array}{c}0.87 \pm 0.15 \\
(0.80-0.94)\end{array}$ & $\begin{array}{c}0.69 \pm 0.14 \\
(0.62-0.76)\end{array}$ & $\begin{array}{c}0.57 \pm 0.09 \\
(0.53-0.61)\end{array}$ & $\begin{array}{c}0.42 \pm 0.09 \\
(0.38-0.46)\end{array}$ & $\begin{array}{c}0.27 \pm 0.08 \\
(0.23-0.31)\end{array}$ & \\
\hline
\end{tabular}

Results are mean $\pm S D$ (90\% confidence intervals). 1RM, 1 repetition maximum; NO-BFR, no blood flow restriction (control); I-BFR, intermittent blood flow restriction; C-BFR, continuous blood flow restriction.

as may be the case in heavier loads, then an additional BFR by the cuffs would not cause any further metabolic effects, explaining the similar performance in all three conditions when the load was $\geq 60 \% 1 \mathrm{RM}$. In contrast, when the loads are very low, there may be less blood flow decrease due to muscle contraction and less muscle swelling during recovery, and thus the effects of external pressure may differentiate the metabolic responses in both I-BFR and C-BFR, compared with the control condition. In a previous study (Wilk et al., 2020f), it was shown that at higher resistive loads (70\% 1RM) a higher occlusion pressure and a wider cuff are necessary to achieve an increase in bar velocity during exercise under BFR. Therefore, it can be speculated that when the external load increases in order to induce greater bar velocity under BFR, a simultaneous increase in pressure or width of the cuff must occur or both at the same time. Therefore, it may be suggested that there may be an interaction between resistive load, cuff width and cuff pressure, which determines the magnitude of acute changes in performance during exercise under BFR, which is also consistent with previous studies (Loenneke et al., 2012b; Rossow et al., 2012).

A possible explanation for the increases in $\mathrm{PV}$ of the bar for both I-BFR and C-BFR, may be ischemic preconditioning (de Souza et al., 2019). Ischemic preconditioning is a noninvasive technique inducing transient peripheral hypoxia and subsequently enhancing tissue tolerance against ischemiareperfusion (Paradis-Deschênes et al., 2016). The ischemic preconditioning technique has been suggested as a potential ergogenic aid to improve exercise performance (Incognito et al., 2016; Marocolo et al., 2016, 2017). The hyperemia experienced following occlusion is related to increased nitric oxide production (Singh et al., 2017). Furthermore, an increased phosphocreatine resynthesis, altered oxy-deoxyhemoglobin kinetics (Bailey et al., 2012), and increased oxygen consumption (Andreas et al., 2011) following brief ischemia, may all play a significant role in improving subsequent exercise performance. However, the effects of previous ischemia would be expected to be enhanced in each subsequent set, which was not the case in the present study, as improvements in PV were observed in only the first four loads. An additional factor which may influence peak performance in both I-BFR and C-BFR may be related with the bidirectional brain-body integration mechanism, which may promote physiological responses through mechanical-sensory receptors (Taylor et al., 2010; Cromwell and Panksepp, 2011; de Souza et al., 2019) thus increasing resistance exercise performance (de Souza et al., 2019). Such physiological responses may potentially explain the significant increase in PV for conditions under BFR compared to NO-BFR.

The most likely factor that may have enhanced PV in the present study in both BFR conditions is the mechanical energy generated by the cuff (Rawska et al., 2019; Wilk et al., 2020d,f). A cuff is a passive element, but during the eccentric phase of the movement, the strain of the material of the cuff, can store and return elastic energy during the concentric phase of the movement (Harman and Frykman, 1990; Wilk et al., 2020f). 


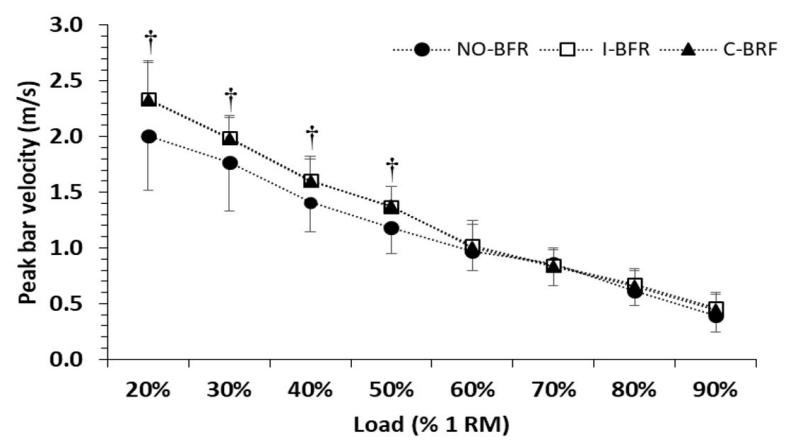

FIGURE 2 | Peak bar velocity during the bench press exercise for eight different loads. 1RM, 1 repetition maximum; NO-BFR, no blood flow restriction (control); I-BFR, intermittent blood flow restriction; C-BFR, continuous blood flow restriction.

This effect may be similar with the phenomenon of enhanced mechanical energy when compressive gear (special shirt for example Inzer or Titan) is used during bench press or powerlifting competitions (Harman and Frykman, 1990), where the energy stored in these elements during the eccentric phase is returned during the lifting phase, resulting in a "rebound" effect. This may be a possible explanation for the increases in PV, but not $\mathrm{MV}$, during fast movements against lower resistive loads. When the load is higher, movement is much slower (see Figure 2), and therefore a wider and highly pressurized cuff which may store much more energy, is needed to induce performance changes (Wilk et al., 2020f). Confirmation of the importance of cuff width and cuff pressure in order to induce performance changes has been provided in the study of Wilk et al. (2020d), who showed a significant acute increase in maximum strength in BP when a wide cuff with pressure of $150 \%$ AOP, but not with $100 \%$ AOP, was used for BFR.

In the present study there were no significant differences between I-BFR and C-BFR conditions regarding performance enhancement (Figure 2 and Tables 1, 2). The lack of differences between the I-BFR and C-BFR is surprising, since there was a large difference in duration of time under occlusion between both conditions. The occlusion during the BP with C-BFR lasted approximately $23 \mathrm{~min}$ (eight sets, $3 \mathrm{~min}$ rest intervals, $\sim 5$ s of effort for each set), however, during I-BFR it was only approximately $40 \mathrm{~s}$ (eight sets, $\sim 5 \mathrm{~s}$ of effort for each set). Previous studies showed that C-BFR leads to the immediate onset of physiological and metabolic stress (Neto et al., 2018; Wilk et al., 2018; Okita et al., 2019), which consequently may lead to increased fatigue and decreased exercise performance compared to NO-BFR condition (Wernbom et al., 2009; Loenneke et al., 2012a). However, it is expected that due to the brief duration of occlusion the I-BFR, metabolic disturbances would be much lower compared to C-BFR (Pearson and Hussain, 2015; Teixeira E. L. et al., 2018; Okita et al., 2019). Thus, despite the possible differences in metabolic stress between I-BFR and C-BFR, changes in PV were identical, suggesting that enhancement of PV should be attributed to mechanical rather to physiological factors.

Despite the greater metabolic stress potentially induced by C-BFR (Okita et al., 2019), the results did not show any negative effects on $\mathrm{PV}$ and $\mathrm{MV}$ at any load, compared with NO-BFR (Figure 2 and Tables 1, 2). This may be caused by the short duration of each set (two repetitions lasting approximately $5 \mathrm{~s}$ ) and the fact that such brief efforts are fueled mainly by phosphocreatine (Bird et al., 2005), which is largely restored during short rest intervals (Bogdanis et al., 1996; Dawson et al., 2007). Performing repetitions to exhaustion at each load would be expected to cause significant reductions in exercise capacity under BFR (Wernbom et al., 2009). In contrast, peak performance is either enhanced at lower loads, or remains unaffected by BFR at higher loads. However, attention should be paid not only to the direct effect of BFR on exercise capacity but also on the potential post-exercise physiological consequences, such as possible muscle damage or even rhabdomyolysis (Wernbom et al., 2019, 2020). Exerciseinduced muscle damage is typically caused by unaccustomed eccentric exercise with high load, while BFR in combination with resistance exercise may cause muscle damage, even when exercising against low loads to volitional exhaustion for multiple sets (Yasuda et al., 2015; Sieljacks et al., 2016). Possible muscle damage after exercise under BFR will affect the process of recovery and may affect the quality of the training sessions that follow. Unfortunately, in the present study we did not assess muscle damage indices in the days following the exercise sessions, since this was not within the scope of this research. However, load volume was low, (eight sets of two repetitions each) and exercise was not exhaustive, as the recovery interval was full (3 min). Moreover, our subjects did not report any signs of muscle damage, such as delayed onset muscle soreness. Nevertheless, the C-BFR condition, where blood flow is restricted for $23 \mathrm{~min}$, may result in muscle damage even with this relatively low volume workout (Wernbom et al., 2019, 2020). Thus, the I-BFR seems more attractive in order achieve improved bench press performance and thus training load, while minimizing the negative effects of BFR.

Although the results of the present study showed that both intermittent and continuous BFR during resistance exercise may be used to enhance peak performance during upper body resistance exercises, there are certain limitations which should be addressed. Although the results showed that both I-BFR as well C-BFR impact bar velocity, during the BP at light and moderate loads, the causes of these changes could not be determined and explained due to the lack of physiological and biomechanical evaluations, which could provide possible explanations. Furthermore, the results of this study may not translate to other types of exercises, different loads or different cuff pressures, and thus further research is required.

\section{Practical Implications}

The present study showed that resistance exercise under narrow BFR can be effectively used to increase bar velocity during resistance exercises of the upper body. However, the observed increases in bar velocity apply only to peak values and light to moderate external loads. The applied BFR techniques using a narrow cuff, do not seem to improve MV of the bar and are not effective with loads over 50\% 1RM. As previously shown, greater external loads require a higher pressure or wider cuffs 
to induce a more severe metabolic stress and increase bar velocity. Furthermore, despite the lack of an increase in PV following BFR at higher loads it should be indicated that C-BFR during resistance training could induce additional physiological responses (not evaluated in the present study), such as metabolic stress, endocrine responses, reinforcement of intramuscular signaling, and increased recruitment of fast twitch muscle fibers. Therefore, maintaining mean and peak bar velocity while increasing physiological responses during resistance exercise under BFR, can be a significant factor determining the level of post-exercise adaptive changes. Despite the beneficial effect of BFR on acute bar velocity changes, occlusion can also cause several side effects, especially when used too frequently. The possibility of muscle damage following this protocol cannot be excluded, especially in the C-BFR condition, where blood flow is restricted for a long time. Thus, the I-BFR may be preferable in order to maximize training effects, without the negative effects of BFR.

Further the disadvantages relate primarily to the weakening of the musculature in the area of direct application of the occlusive cuff, especially when the cuff is narrow (Kacin and Strazar, 2011; Ellefsen et al., 2015). Therefore, therapists and trainers should introduce BFR protocols carefully and gradually progress them over time, to ensure that protective adaptations (i.e., a repeated bout effect) can take place in order to minimize the risk of excessive muscle stress and damage (Clark and Manini, 2017; Wernbom et al., 2019). Also, the intermittent form of BFR may be preferable. Therefore, the use of BFR during resistance exercise should be used as a supplementary and not a main method to enhance adaptive responses. It seems that a combination of heavy loaded traditional resistance exercise and supplementary exercise under BFR provide the most desired adaptive changes.

\section{CONCLUSION}

The results of the present study indicated that BFR used during resistance exercise increases peak bar velocity and thus can by useful for improving explosive performance during resistance

\section{REFERENCES}

Andreas, M., Schmid, A. I., Keilani, M., Doberer, D., Bartko, J., Crevenna, R., et al. (2011). Effect of ischemic preconditioning in skeletal muscle measured by functional magnetic resonance imaging and spectroscopy: a randomized crossover trial. J. Cardiovasc. Magn. Reson. 13:32. doi: 10.1186/1532-429X13-32

Bailey, T. G., Birk, G. K., Cable, N. T., Atkinson, G., Green, D. J., Jones, H., et al. (2012). Remote ischemic preconditioning prevents reduction in brachial artery flow-mediated dilation after strenuous exercise. Am. J. Physiol.-Heart Circ. Physiol. 303, H533-H538. doi: 10.1152/ajpheart.00272.2012

Bell, Z. W., Abe, T., Wong, V., Spitz, R. W., Viana, R. B., Chatakondi, R. N., et al. (2020). Muscle swelling following blood flow-restricted exercise does not differ between cuff widths in the proximal or distal portions of the upper leg. Clin. Physiol. Funct. Imaging 40, 269-276. doi: 10.1111/cpf.12635

Bird, S. P., Tarpenning, K. M., and Marino, F. E. (2005). Designing resistance training programmes to enhance muscular fitness: a review of the acute programme variables. Sports Med. 35, 841-851. doi: 10.2165/00007256200535100-00002 training. However, such improvements under BFR were observed only at loads from 20 to 50\% 1RM and did not carry over to higher loads. Peak velocity enhancement under BFR may be explained by both metabolic and mechanical factors, with the latter possibly being more influential. These findings expand the scientific knowledge related to the acute effects of BFR during resistance exercise, which also has significant practical implications.

\section{DATA AVAILABILITY STATEMENT}

The raw data supporting the conclusions of this article will be made available by the authors, without undue reservation.

\section{ETHICS STATEMENT}

The studies involving human participants were reviewed and approved by The study protocol was approved by the Bioethics Committee for Scientific Research, at the Academy of Physical Education in Katowice, Poland (02/2019). The patients/participants provided their written informed consent to participate in this study.

\section{AUTHOR CONTRIBUTIONS}

MW, MG, PS, and MK: study conception and design. MW and MG: acquisition of data. MW, MK, and PS: analysis and interpretation of data. MW, MG, GB, and PS: drafting of manuscript. MW, PS, AZ, and GB: critical revision. All authors contributed to the article and approved the submitted version.

\section{FUNDING}

The study was supported and funded by the statutory research of the Jerzy Kukuczka Academy of Physical Education in Katowice, Poland and by Charles University grant UNCE/HUM/032.

Bogdanis, G. C., Nevill, M. E., Boobis, L. H., and Lakomy, H. K. (1996). Contribution of phosphocreatine and aerobic metabolism to energy supply during repeated sprint exercise. J. Appl. Physiol. 80, 876-884. doi: 10.1152/jappl. 1996.80.3.876

Clark, B. C., and Manini, T. M. (2017). Can KAATSU exercise cause rhabdomyolysis? Clin. J. Sport Med. 27, e1-e2. doi: 10.1097/JSM. 0000000000000309

Cohen, J. (1988). Statistical Power Analysis for the Behavioral Sciences, 2 Edn. Hillsdale, NJ: L. Erlbaum Associates.

Cromwell, H. C., and Panksepp, J. (2011). Rethinking the cognitive revolution from a neural perspective: how overuse/misuse of the term 'cognition' and the neglect of affective controls in behavioral neuroscience could be delaying progress in understanding the BrainMind. Neurosci. Biobehav. Rev. 35, 2026-2035. doi: 10.1016/j.neubiorev.2011. 02.008

Dawson, B., Goodman, C., Lawrence, S., Preen, D., Polglaze, T., Fitzsimons, M., et al. (2007). Muscle phosphocreatine repletion following single and repeated short sprint efforts. Scand. J. Med. Sci. Sports 7, 206-213. doi: 10.1111/j.16000838.1997.tb00141.x 
de Souza, H. L. R., Arriel, R. A., Hohl, R., da Mota, G. R., and Marocolo, M. (2019). Is ischemic preconditioning intervention occlusion-dependent to enhance resistance exercise performance?. J. Strength Cond. Res. 1, doi: 10.1519/ JSC.0000000000003224 [Online ahead of print]

Dunnick, D. D., Brown, L. E., Coburn, J. W., Lynn, S. K., and Barillas, S. R. (2015). Bench Press Upper-Body Muscle Activation Between Stable and Unstable Loads. J. Strength Cond. Res. 29, 3279-3283. doi: 10.1519/JSC. 0000000000001198

Ellefsen, S., Hammarström, D., Strand, T. A., Zacharoff, E., Whist, J. E., Rauk, I., et al. (2015). Blood flow-restricted strength training displays high functional and biological efficacy in women: a within-subject comparison with high-load strength training. Am. J. Physiol.-Regul. Integr. Comp. Physiol. 309, R767-R779. doi: 10.1152/ajpregu.00497.2014

Garnacho-Castaño, M. V., López-Lastra, S., and Maté-Muñoz, J. L. (2015). Reliability and validity assessment of a linear position transducer. J. Sports Sci. Med. 14, 128-136.

Golas, A., Maszczyk, A., Wilk, M., and Stastny, P. (2015). Changes in bar velocity and muscular activity during the bench press in relation to the load lifted. Cent. Eur. J. Sport Sci. Med. 11, 95-101. doi: 10.18276/cej.2015.3-11

Harman, E., and Frykman, P. (1990). The effects of knee wraps on weightlifting per- formance and injury. J. Strength Cond. Res. 12, 30-35. doi: 10.1519/07440049(1990)012<0030:teokwo>2.3.co;2

Incognito, A. V., Burr, J. F., and Millar, P. J. (2016). The effects of ischemic preconditioning on human exercise performance. Sports Med. 46, 531-544. doi: 10.1007/s40279-015-0433-5

Jessee, M. B., Buckner, S. L., Dankel, S. J., Counts, B. R., Abe, T., and Loenneke, J. P. (2016). The influence of cuff width, sex, and race on arterial occlusion: implications for blood flow restriction research. Sports Med. 46, 913-921. doi: 10.1007/s40279-016-0473-5

Kacin, A., and Strazar, K. (2011). Frequent low-load ischemic resistance exercise to failure enhances muscle oxygen delivery and endurance capacity: ischemic training and muscle endurance. Scand. J. Med. Sci. Sports 21, e231-e241. doi: 10.1111/j.1600-0838.2010.01260.x

Krzysztofik, M., Wilk, M., Wojdala, G., and Golas, A. (2019). Maximizing muscle hypertrophy: a systematic review of advanced resistance training techniques and methods. Int. J. Environ. Res. Public. Health 16:4897. doi: 10.3390/ ijerph 16244897

Loenneke, J., Fahs, C., Thiebaud, R., Rossow, L., Abe, T., Ye, X., et al. (2012). The acute muscle swelling effects of blood flow restriction. Acta Physiol. Hung. 99, 400-410. doi: 10.1556/APhysiol.99.2012.4.4

Loenneke, J. P., Balapur, A., Thrower, A. D., Barnes, J., and Pujol, T. J. (2012a). Blood flow restriction reduces time to muscular failure. Eur. J. Sport Sci. 12, 238-243. doi: 10.1080/17461391.2010.551420

Loenneke, J. P., Fahs, C. A., Rossow, L. M., Sherk, V. D., Thiebaud, R. S., Abe, T., et al. (2012b). Effects of cuff width on arterial occlusion: implications for blood flow restricted exercise. Eur. J. Appl. Physiol. 112, 2903-2912. doi: 10. 1007/s00421-011-2266-2268

Loenneke, J. P., Allen, K. M., Mouser, J. G., Thiebaud, R. S., Kim, D., Abe, T., et al. (2015). Blood flow restriction in the upper and lower limbs is predicted by limb circumference and systolic blood pressure. Eur. J. Appl. Physiol. 115, 397-405. doi: 10.1007/s00421-014-3030-3037

Loenneke, J. P., and Pujol, T. J. (2009). The use of occlusion training to produce muscle hypertrophy. Strength Cond. J. 31, 77-84. doi: 10.1519/SSC. 0b013e3181a5a352

Luebbers, P. E., Fry, A. C., Kriley, L. M., and Butler, M. S. (2014). The effects of a 7-week practical blood flow restriction program on well-trained collegiate athletes. J. Strength Cond. Res. 28, 2270-2280. doi: 10.1519/JSC. 0000000000000385

Marocolo, I. C., da Mota, G. R., Londe, A. M., Patterson, S. D., Barbosa Neto, O., and Marocolo, M. (2017). Acute ischemic preconditioning does not influence high-intensity intermittent exercise performance. PeerJ 5:e4118. doi: 10.7717/ peerj. 4118

Marocolo, M., Billaut, F., and da Mota, G. R. (2018). Ischemic preconditioning and exercise performance: an ergogenic aid for whom? Front. Physiol. 9:1874. doi: $10.3389 /$ fphys.2018.01874

Marocolo, M., Simim, M. A. M., Bernardino, A., Monteiro, I. R., Patterson, S. D., and da Mota, G. R. (2019). Ischemic preconditioning and exercise performance: shedding light through smallest worthwhile change. Eur. J. Appl. Physiol. 119, 2123-2149. doi: 10.1007/s00421-019-04214-4216
Marocolo, M., Willardson, J. M., Marocolo, I. C., da Mota, G. R., Simão, R., and Maior, A. S. (2016). Ischemic preconditioning and placebo intervention improves resistance exercise performance. J. Strength Cond. Res. 30, 1462-1469. doi: 10.1519/JSC.0000000000001232

Neto, G. R., Novaes, J. S., Salerno, V. P., Gonçalves, M. M., Batista, G. R., and Cirilo-Sousa, M. S. (2018). Does a resistance exercise session with continuous or intermittent blood flow restriction promote muscle damage and increase oxidative stress? J. Sports Sci. 36, 104-110. doi: 10.1080/02640414.2017.128 3430

Okita, K., Takada, S., Morita, N., Takahashi, M., Hirabayashi, K., Yokota, T., et al. (2019). Resistance training with interval blood flow restriction effectively enhances intramuscular metabolic stress with less ischemic duration and discomfort. Appl. Physiol. Nutr. Metab. 44, 759-764. doi: 10.1139/apnm-20182321

Paradis-Deschênes, P., Joanisse, D. R., and Billaut, F. (2016). Ischemic preconditioning increases muscle perfusion, oxygen uptake, and force in strength-trained athletes. Appl. Physiol. Nutr. Metab. 41, 938-944. doi: 10.1139/ apnm-2015-2561

Pearson, S. J., and Hussain, S. R. (2015). A review on the mechanisms of bloodflow restriction resistance training-induced muscle hypertrophy. Sports Med. 45, 187-200. doi: 10.1007/s40279-014-0264-9

Ploutz-Snyder, L. L., Convertino, V. A., and Dudley, G. A. (1995). Resistance exercise-induced fluid shifts: change in active muscle size and plasma volume. Am. J. Physiol.-Regul. Integr. Comp. Physiol. 269, R536-R543. doi: 10.1152/ ajpregu.1995.269.3.R536

Rawska, M., Gepfert, M., Mostowik, A., Krzysztofik, M., Wojdala, G., Lulinska, A., et al. (2019). Does blood flow restriction influence the maximal number of repetitions performed during the bench press? A pilot study. Balt. J. Health Phys. Act. 11, 9-17. doi: 10.29359/BJHPA.11.4.02

Rossow, L. M., Fahs, C. A., Loenneke, J. P., Thiebaud, R. S., Sherk, V. D., Abe, T., et al. (2012). Cardiovascular and perceptual responses to blood-flow-restricted resistance exercise with differing restrictive cuffs. Clin. Physiol. Funct. Imaging 32, 331-337. doi: 10.1111/j.1475-097X.2012.01131.x

Scott, B. R., Loenneke, J. P., Slattery, K. M., and Dascombe, B. J. (2015). Exercise with blood flow restriction: an updated evidence-based approach for enhanced muscular development. Sports Med. 45, 313-325. doi: 10.1007/s40279-0140288-1

Shimizu, R., Hotta, K., Yamamoto, S., Matsumoto, T., Kamiya, K., Kato, M., et al. (2016). Low-intensity resistance training with blood flow restriction improves vascular endothelial function and peripheral blood circulation in healthy elderly people. Eur. J. Appl. Physiol. 116, 749-757. doi: 10.1007/s00421-016-33283328

Sieljacks, P., Matzon, A., Wernbom, M., Ringgaard, S., Vissing, K., and Overgaard, K. (2016). Muscle damage and repeated bout effect following blood flow restricted exercise. Eur. J. Appl. Physiol. 116, 513-525. doi: 10.1007/s00421-0153304-8

Singh, L., Randhawa, P. K., Singh, N., and Jaggi, A. S. (2017). Redox signaling in remote ischemic preconditioning-induced cardioprotection: evidences and mechanisms. Eur. J. Pharmacol. 809, 151-155. doi: 10.1016/j.ejphar.2017. 05.033

Sjøgaard, G., Savard, G., and Juel, C. (1988). Muscle blood flow during isometric activity and its relation to muscle fatigue. Eur. J. Appl. Physiol. Occup. Physiol. 57, 327-335. doi: 10.1007/BF00635992

Stastny, P., Golas, A., Blazek, D., Maszczyk, A., Wilk, M., Pietraszewski, P., et al. (2017). A systematic review of surface electromyography analyses of the bench press movement task. PLoS One 12:e0171632. doi: 10.1371/journal.pone. 0171632

Takano, H., Morita, T., Iida, H., Asada, K., Kato, M., Uno, K., et al. (2005). Hemodynamic and hormonal responses to a short-term low-intensity resistance exercise with the reduction of muscle blood flow. Eur. J. Appl. Physiol. 95, 65-73. doi: 10.1007/s00421-005-1389-1381

Taylor, A. G., Goehler, L. E., Galper, D. I., Innes, K. E., and Bourguignon, C. (2010). Top-Down and bottom-up mechanisms in mind-body medicine: development of an integrative framework for psychophysiological research. Explore 6, 29-41. doi: 10.1016/j.explore.2009.10.004

Teixeira, E. L., Barroso, R., Silva-Batista, C., Laurentino, G. C., Loenneke, J. P., Roschel, H., et al. (2018). Blood flow restriction increases metabolic stress but decreases muscle activation during high-load resistance exercise. Muscle Nerve 57, 107-111. doi: 10.1002/mus.25616 
Teixeira, V., Voci, S. M., Mendes-Netto, R. S., and da Silva, D. G. (2018). The relative validity of a food record using the smartphone application MyFitnessPal: relative validity of a smartphone dietary record. Nutr. Diet. 370, 219-225. doi: 10.1111/1747-0080.12401

Wernbom, M., Järrebring, R., Andreasson, M. A., and Augustsson, J. (2009). Acute effects of blood flow restriction on muscle activity and endurance during fatiguing dynamic knee extensions at low load. J. Strength Cond. Res. 23, 2389-2395. doi: 10.1519/JSC.0b013e3181bc1c2a

Wernbom, M., Paulsen, G., Bjørnsen, T., Cumming, K., and Raastad, T. (2019). Risk of muscle damage with blood flow-restricted exercise should not be overlooked. Clin. J. Sport Med. doi: 10.1097/JSM.0000000000000755 [Epubh ahead of print].

Wernbom, M., Schoenfeld, B. J., Paulsen, G., Bjørnsen, T., Cumming, K. T., Aagaard, P., et al. (2020). Commentary: can blood flow restricted exercise cause muscle damage? Commentary on blood flow restriction exercise: considerations of methodology, application, and safety. Front. Physiol. 11:243. doi: 10.3389/ fphys.2020.00243

Wilk, M., Gepfert, M., Krzysztofik, M., Golas, A., Mostowik, A., Maszczyk, A., et al. (2019). The influence of grip width on training volume during the bench press with different movement tempos. J. Hum. Kinet. 68, 49-57. doi: 10.2478/hukin2019-2055

Wilk, M., Gepfert, M., Krzysztofik, M., Mostowik, A., Filip, A., Hajduk, G., et al. (2020a). Impact of duration of eccentric movement in the one-repetition maximum test result in the bench press among women. J. Sports Sci. Med. 19, 317-322.

Wilk, M., Golas, A., Zmijewski, P., Krzysztofik, M., Filip, A., Coso, J. D., et al. (2020b). The effects of the movement tempo on the one-repetition maximum bench press results. J. Hum. Kinet. 72, 151-159. doi: 10.2478/hukin-2020-2021

Wilk, M., Krzysztofik, M., Drozd, M., and Zajac, A. (2020c). Changes of power output and velocity during successive sets of the bench press with different duration of eccentric movement. Int. J. Sports Physiol. Perform. 15, 162-167. doi: 10.1123/ijspp.2019-2164
Wilk, M., Krzysztofik, M., Filip, A., Lockie, R. G., and Zajac, A. (2020d). The acute effects of external compression with blood flow restriction on maximal strength and strength-endurance performance of the upper limbs. Front. Physiol. doi: 10.3389/fphys.2020.00567 [Epub ahead of print],

Wilk, M., Krzysztofik, M., Filip, A., Szkudlarek, A., Lockie, R. G., and Zajac, A. (2020e). Does post-activation performance enhancement occur during the bench press exercise under blood flow restriction? Int. J. Environ. Res. Public. Health 17:3752. doi: 10.3390/ijerph17113752

Wilk, M., Krzysztofik, M., Filip, A., Zajac, A., Bogdanis, G. C., and Lockie, R. G. (2020f). Short-term blood flow restriction increases power output and bar velocity during the bench press. J. Strength Cond. Res. doi: 10.1519/JSC. 0000000000003649 [Online ahead of print]

Wilk, M., Krzysztofik, M., Gepfert, M., Poprzecki, S., Golas, A., and Maszczyk, A. (2018). Technical and training related aspects of resistance training using blood flow restriction in competitive sport - a review. J. Hum. Kinet. 65, 249-260. doi: 10.2478/hukin-2018-2101

Yasuda, T., Fukumura, K., Iida, H., and Nakajima, T. (2015). Effect of low-load resistance exercise with and without blood flow restriction to volitional fatigue on muscle swelling. Eur. J. Appl. Physiol. 115, 919-926. doi: 10.1007/s00421014-3073-9

Conflict of Interest: The authors declare that the research was conducted in the absence of any commercial or financial relationships that could be construed as a potential conflict of interest.

Copyright (c) 2020 Wilk, Gepfert, Krzysztofik, Stastny, Zajac and Bogdanis. This is an open-access article distributed under the terms of the Creative Commons Attribution License (CC BY). The use, distribution or reproduction in other forums is permitted, provided the original author(s) and the copyright owner(s) are credited and that the original publication in this journal is cited, in accordance with accepted academic practice. No use, distribution or reproduction is permitted which does not comply with these terms. 